\title{
Moments of Dual Generalized Order Statistics from Inverted Kumaraswamy Distribution and Related Inference
}

\author{
Bushra Khatoon, M. J. S. Khan ${ }^{\star}$, Zubdahe Noor \\ Department of Statistics and Operations Research, Aligarh Muslim University, Aligarh, 202 002, India
}

\section{ARTICLE INFO}

\section{Article History}

Received 12 Mar 2020

Accepted 24 Nov 2020

\section{Keywords}

Moments

Dual generalized order statistics

Maximum likelihood estimation

Uniformly minimum variance

unbiased estimation

AMS Subject Classification: $62 \mathrm{G} 30,62 \mathrm{E} 15,62 \mathrm{E} 10,60 \mathrm{E} 05$.

\begin{abstract}
AB S TRACT
In this paper, the exact and explicit expressions for single, product, and conditional moments for inverted Kumaraswamy distribution using dual generalized order statistics (dgos) are derived. Further, the expression for maximum likelihood estimator (MLE) and uniformly minimum variance unbiased estimator (UMVUE) for the parameters of inverted Kumaraswamy distribution based on dgos are deduced. Also, we obtained the results for order statistics and lower record values by putting some specific values of the parameters of dgos. Finally, a simulation study is carried out for illustrative purpose.
\end{abstract}

(C) 2021 The Authors. Published by Atlantis Press B.V. This is an open access article distributed under the CC BY-NC 4.0 license (http://creativecommons.org/licenses/by-nc/4.0/).

\section{INTRODUCTION}

Generalized order statistics (gos) was introduced by Kamps [1] as a unified model of ordered random variables arranged in increasing order of magnitude which contains almost all models related to ordered random variables such as order statistics, records, Pfeifer records, progressive type-II censored order statistics, and sequential order statistics. But those models of ordered random variables which are in decreasing order of magnitude cannot be studied in this framework. To resolve such types of problems, Pawlas and Szynal [2] proposed the concept of dual (lower) generalized order statistics (dgos) which contains several models of ordered random variables those are arranged in decreasing order of magnitude, like reversed order statistics, lower records, and lower Pfeifer records. Burkschat et al. [3] established the direct relationship between gos and dgos in such a way that if $X(1, n, \tilde{m}, k), X(2, n, \tilde{m}, k), \ldots, X(n, n, \tilde{m}, k)$ are $n$ gos with cumulative distribution function $(c d f) F_{0}($.$) and X_{d}(1, n, \tilde{m}, k), X_{d}(2, n, \tilde{m}, k), \ldots, X_{d}(n, n, \tilde{m}, k)$ are $n$ dgos with $(c d f) F($.$) , then$

$$
F\left(X_{d}(i, n, \tilde{m}, k)\right) \stackrel{d}{=} 1-F_{0}(X(i, n, \tilde{m}, k)), \quad 1 \leq i \leq n
$$

where $\stackrel{d}{=}$ denotes the convergence in distribution.

Therefore, to avoid the complications in calculations, one can use dgos when $F($.$) is available in compact and closed form and when a closed$ and compact form of $(1-F()$.$) is available, gos can be preferred.$

Let $X_{1}, X_{2}, \ldots, X_{n}$ be a sequence of independent and identically distributed (iid) random variables with absolutely continuous distribution function $(d f) F(x)$ and the probability density function $(p d f) f(x), x \in(-\infty, \infty)$. Further, let $n \in \mathbb{N}, n \geq 2, k \geq 1, \tilde{m}=\left(m_{1}, m_{2}, \ldots, m_{n-1}\right) \in$ $\mathbb{R}^{n-1}, M_{r}=\sum_{j=r}^{n-1} m_{j}$, such that $\gamma_{r}=k+n-r+M_{r}>0$ for all $r \in\{1,2, \ldots, n-1\}$. Then $X_{d}(r, n, \tilde{m}, k), r=1,2, \ldots, n$ are said to be $d g o s$ if their joint $p d f$ is given by

$$
k\left(\prod_{j=1}^{n-1} \gamma_{j}\right)\left(\prod_{i=1}^{n-1}\left[F\left(x_{i}\right)\right]^{m_{i}} f\left(x_{i}\right)\right)\left[F\left(x_{n}\right)\right]^{k-1} f\left(x_{n}\right)
$$

for $F^{-1}(1)>x_{1} \geq x_{2} \geq \ldots \geq x_{n}>F^{-1}(0)$. 
Note that $X_{d}(r, n, \tilde{m}, k)$ reduces to the $(n-j+1)^{\text {th }}$ order statistics from a sample of size $n$ if $\gamma_{j}=n-j+1\left(i . e \cdot m_{1}=m_{2}=\ldots=m_{n-1}=0\right.$ and $k=1)$ and when $m \rightarrow-1, k=1, X_{d}(r, n, \tilde{m}, k)$ reduces to $r^{\text {th }}$ lower records.

Here, we shall consider two cases:

Case I: $\gamma_{i} \neq \gamma_{j}, i \neq j$ i.e. $\gamma_{i}$ 's are pairwise different.

The $p d f$ of $r^{\text {th }} d g o s X_{d}(r, n, \tilde{m}, k)$ is given by (Kamps [1])

$$
f_{X_{d}(r, n, \tilde{m}, k)}(x)=c_{r-1} \sum_{i=1}^{r} a_{i}(r)[F(x)]^{\gamma_{i}-1} f(x), \quad-\infty<x<\infty
$$

And the joint $p d f$ of $X_{d}(r, n, \tilde{m}, k)$ and $X_{d}(s, n, \tilde{m}, k)$ is:

$$
\begin{aligned}
f_{X_{d}(r, n, \tilde{m}, k), X_{d}(s, n, \tilde{m}, k)}(x, y)= & c_{s-1} \sum_{i=1}^{r} \sum_{j=r+1}^{s} a_{i}(r) a_{j}^{(r)}(s)\left[\frac{F(y)}{F(x)}\right]^{\gamma_{j}}[F(x)]^{\gamma_{i}} \\
& \times \frac{f(x)}{F(x)} \frac{f(y)}{F(y)}, \quad-\infty<y<x<\infty
\end{aligned}
$$

Therefore, the conditional $p d f$ of $X_{d}(s, n, \tilde{m}, k)$ given $X_{d}(r, n, \tilde{m}, k)=x$, for $1 \leq r<s \leq n$ is:

$$
f_{X_{d}(s, n, \tilde{m}, k) \mid X_{d}(r, n, \tilde{m}, k)}(y \mid x)=\frac{c_{s-1}}{c_{r-1}} \sum_{j=r+1}^{s} a_{j}^{(r)}(s)\left[\frac{F(y)}{F(x)}\right]^{\gamma_{j}} \frac{f(y)}{F(y)}, \quad-\infty<y<x<\infty
$$

similarly, the conditional $p d f$ of $X_{d}(r, n, \tilde{m}, k) \mid X_{(s, n, \tilde{m}, k)}=y, 1 \leq r<s \leq n$, is given by

$$
f_{X_{d}(r, n, \tilde{m}, k) \mid X_{d}(s, n, \tilde{m}, k)}(x \mid y)=\frac{\sum_{i=1}^{r} \sum_{j=r+1}^{s} a_{j}^{(r)}(s) a_{i}(r)\left[\frac{F(y)}{F(x)}\right]^{\gamma_{j}}[F(x)]^{\gamma_{i}} \frac{f(x)}{F(x)}}{\sum_{t=1}^{s} a_{t}(s)[F(y)]^{\gamma_{t}}},-\infty<y<x<\infty
$$

where

$$
a_{i}(r)=\prod_{\substack{j=1 \\ j \neq i}}^{r} \frac{1}{\left(\gamma_{j}-\gamma_{i}\right)}, \quad \gamma_{i} \neq \gamma_{j}, 1 \leq i \leq r \leq n
$$

and

$$
a_{i}^{(r)}(s)=\prod_{\substack{j=r+1 \\ j \neq i}}^{s} \frac{1}{\left(\gamma_{j}-\gamma_{i}\right)}, \quad \gamma_{i} \neq \gamma_{j}, r+1 \leq i \leq s \leq n
$$

It may be noted that for $m_{1}=\ldots=m_{n-1}=m \neq-1,($ Khan and Khan [4])

$$
\begin{gathered}
a_{i}(r)=\frac{(-1)^{r-i}}{(m+1)^{s-r-1}(r-1) !}\left(\begin{array}{c}
r-1 \\
i
\end{array}\right) \\
a_{i}^{r}(s)=\frac{(-1)^{r-i}}{(m+1)^{s-r-1}(r-1) !}\left(\begin{array}{c}
s-r-1 \\
i
\end{array}\right)
\end{gathered}
$$


and the joint $p d f$ of $X_{d}(r, n, m, k)$ and $X_{d}(s, n, m, k),(1 \leq r<s \leq n)$ is

$$
\begin{aligned}
f_{X_{d}(r, n, m, k), X_{d}(s, n, m, k)}(x, y)= & \frac{c_{s-1}}{(r-1) !(s-r-1) !}\left[h_{m}^{\prime}(F(y))-h_{m}^{\prime}(F(x))\right]^{s-r-1} g_{m}^{\prime}{ }^{r-1}(F(x)) \\
& \times[F(y)]^{\gamma_{s}-1}[F(x)]^{m} f(x) f(y), \quad-\infty<y<x<\infty
\end{aligned}
$$

where $c_{r-1}=\prod_{i=1}^{r} \gamma_{i}$,

$$
h_{m}^{\prime}(x)= \begin{cases}-\frac{1}{m+1} x^{m+1} & , m \neq-1 \\ -\log x & , m=-1\end{cases}
$$

and $g_{m}^{\prime}(x)=h_{m}^{\prime}(x)-h_{m}^{\prime}(1), x \in(0,1)$.

Further, the conditional $p d f$ of $X_{d}(s, n, m, k)$ given $X_{d}(r, n, m, k)=x, 1 \leq r<s \leq n$ is

$$
\begin{aligned}
f_{X_{d}(s, n, m, k) \mid X_{d}(r, n, m, k)}(y \mid x)= & \frac{c_{s-1}}{c_{r-1}(s-r-1) !}\left[h_{m}^{\prime}(F(y))-h_{m}^{\prime}(F(x))\right]^{s-r-1} \\
& \times \frac{[F(y)]^{\gamma_{s}-1}}{[F(x)]^{\gamma_{r+1}}} f(y), \quad-\infty<y<x<\infty
\end{aligned}
$$

And the conditional $p d f$ of $X_{d}(r, n, m, k)$ given $X_{d}(s, n, m, k)=x, 1 \leq r<s \leq n$ is

$$
\begin{aligned}
f_{X_{d}(r, n, m, k) \mid X_{d}(s, n, m, k)}(x \mid y)= & \frac{(s-1) !}{(r-1) !(s-r-1) !}\left[h_{m}^{\prime}(F(y))-h_{m}^{\prime}(F(x))\right]^{s-r-1} \\
& \times \frac{\left[g_{m}^{\prime}(F(x))\right]^{r-1}}{\left[g_{m}^{\prime}(F(y))\right]^{s-1}}[F(x)]^{m} f(x), \quad-\infty<y<x<\infty
\end{aligned}
$$

Here, the results are derived for case-I as it includes case-II as a particular case of it.

In the last two decades, several researchers have analyzed statistical properties of continuous distributions based on gos, dgos and hence a wide literature is available on the various developments on dgos and gos. Pawlas and Szynal [5] discussed the relation for single and product moments of gos from Pareto, generalized Pareto, and Burr distributions. Cramer et al. [6] proved the existence of moments of gos. Ahsanullah [7] characterized the uniform distribution based on dgos. Athar et al. [8] obtained the explicit expressions for ratio and inverse moments of gos from Weibull distribution. Explicit expressions for single and product moments of gos from linear exponential distribution have been derived by Ahmad [9]. Based on dgos, Khan et al. [10] established the recurrence relations for single and product moments of exponentiated Weibull distribution. Khan et al. [11] have characterized continuous distributions conditioned on a pair of nonadjacent dgos. Burkschat [12] has discussed the linear estimators and predictors from generalized Pareto distribution based on gos. Barakat and Adll [13] studied the asymptotic theory of extreme dgos. Athar and Faizan [14] deduced the explicit expression for moments of power function distribution based on dgos. Khan and Khan [4] obtained the ratio and inverse moments of gos from Burr distribution using hypergeometric functions. Domma and Hamdeni [15] discussed the truncated moments of dgos. Recurrence relations for moments of dgos from Weibull gamma distribution have been discussed by Mahmoud et al. [16]. Recurrence relation for single and product moments of $d g o s$ from a general class of distribution have been derived by Saran et al. [17]. Based on lower records, Khan and Arshad [18] have derived the uniformly minimum variance unbiased estimator(UMVUE) of reliability function and stress-strength reliability from proportional reversed hazard rate model. An exact and explicit expression of moments of Topp-Leone distribution based dgos have been deduced by Khan and Iqrar [19]. They have also derived the expressions for maximum likelihood estimator(MLE) and UMVUE for the parameter of Topp-Leone distribution based on dgos. Interval estimation for Topp-Leone generated family of distributions based on dgos have been considered by Arshad and Jamal [20]. have considered the single, product, and conditional moments of gos as well as parameter estimation based on gos from Kumaraswamy power function distribution.

Kumaraswamy distribution was introduced by Kumaraswamy [21]. This distribution is applicable in many real-life situations and natural phenomenon that are restricted with lower and upper bounds. Over the last decades, there has been a great interest in studying the Kumaraswamy distribution among the researchers. Few of them are Nadarajah [22], Jones [23], Garg [24], Cordeiro and De-Castro [25], Mitnik [26], Safi and Ahmed [27], El-Deen et al. [28]. El-Deen et al. [28] studied the statistical inference for this distribution based on gos. Abd Al-Fattah et al. [29] introduced the inverted Kumaraswamy distribution as the inverted distributions have wide range of applications in real-life situations. Usman and Ahsan-ul-Haque [30], Reyad et al. [31] have studied the generalization form of inverted Kumaraswamy distribution. 
The $p d f$ of inverted Kumaraswamy distribution is given by

$$
f(x ; \alpha, \beta)=\alpha \beta(1+x)^{-(\alpha+1)}\left[1-(1+x)^{-\alpha}\right]^{\beta-1}, \quad x>0, \alpha, \beta>0 .
$$

And its $c d f$ is given by

$$
F(x ; \alpha, \beta)=\left[1-(1+x)^{-\alpha}\right]^{\beta}, \quad x>0, \alpha, \beta>0 .
$$

This paper comprises of 5 sections. In Section 2, the results for single, product, and conditional moments of inverted Kumaraswamy distribution based on dgos have been derived. Section 3 included the theoretical setup and expressions for the MLE of both parameters of inverted Kumaraswamy distribution based on dgos. In Section 4, we have deduced the UMVUE of one shape parameter of inverted Kumaraswamy distribution by assuming that the other shape parameter is known. Finally, a simulation study is being carried out to check the validity and applicability of the derived estimators based on order statistics and lower records which is given in Section 5.

\section{MOMENTS OF dgos}

In this section, we have derived the exact and explicit expressions for single, product, and conditional moments of inverted Kumaraswamy distribution based on dgos. Further, by putting the specific values of the parameters $m$ and $k$, we have deduced single moment, product moment, and conditional moments of order statistics and lower records from inverted Kumaraswamy distribution Table 1-6.

Table 1 Mean of order statistics from inverted Kumaraswamy distribution for $\alpha=5, \beta=0.5,(m=0, k=1)$.

\begin{tabular}{ccccccccc}
\hline $\boldsymbol{n}$ & $\boldsymbol{r}=\mathbf{1}$ & $\boldsymbol{r}=\mathbf{2}$ & $\boldsymbol{r}=\mathbf{3}$ & $\boldsymbol{r}=\mathbf{4}$ & $\boldsymbol{r}=\mathbf{5}$ & $\boldsymbol{r}=\mathbf{6}$ & $\boldsymbol{r}=\mathbf{7}$ & $\boldsymbol{r}=\mathbf{8}$ \\
\hline 1 & 0.1496 & & & & & & & \\
2 & 0.0493 & 0.25 & & & & & & \\
3 & 0.0254 & 0.097 & 0.3265 & & & & & \\
4 & 0.0157 & 0.0546 & 0.1394 & 0.3889 & & & & \\
5 & 0.0108 & 0.0356 & 0.083 & 0.177 & 0.4419 & & & \\
6 & 0.0078 & 0.0253 & 0.0563 & 0.1096 & 0.2107 & 0.4881 & & \\
7 & 0.0060 & 0.0190 & 0.0411 & 0.0765 & 0.1344 & 0.2412 & 0.5292 & \\
8 & 0.0047 & 0.0148 & 0.0315 & 0.0571 & 0.0959 & 0.1576 & 0.2691 & 0.5664 \\
\hline
\end{tabular}

Table 2 Variance of order statistics from inverted Kumaraswamy distribution for $\alpha=5, \beta=0.5,(m=0, k=1)$.

\begin{tabular}{ccccccccc}
\hline $\boldsymbol{n}$ & $\boldsymbol{r}=\mathbf{1}$ & $\boldsymbol{r}=\mathbf{2}$ & $\boldsymbol{r}=\mathbf{3}$ & $\boldsymbol{r}=\mathbf{4}$ & $\boldsymbol{r}=\mathbf{5}$ & $\boldsymbol{r}=\mathbf{6}$ & $\boldsymbol{r}=\mathbf{7}$ & $\boldsymbol{r}=\mathbf{8}$ \\
\hline 1 & 0.06562 & & & & & & & \\
2 & 0.00687 & 0.10420 & & & & & & \\
3 & 0.00195 & 0.01319 & 0.13210 & & & & & \\
4 & 0.00075 & 0.00422 & 0.01867 & 0.15436 & & & & \\
5 & 0.00038 & 0.00183 & 0.00641 & 0.02317 & 0.17302 & & & \\
6 & 0.00021 & 0.00096 & 0.00303 & 0.00849 & 0.02721 & 0.18946 & & \\
7 & 0.00013 & 0.00056 & 0.00161 & 0.00415 & 0.01034 & 0.03072 & 0.20405 & \\
8 & 0.00008 & 0.00035 & 0.00101 & 0.00234 & 0.00520 & 0.01196 & 0.03379 & 0.21729 \\
\hline
\end{tabular}

Table 3 Mean of lower records from inverted Kumaraswamy distribution for $\alpha=2.5: 0.5: 5, \beta=0.5,(m \rightarrow-1, k=1)$.

\begin{tabular}{lcccccc}
\hline & $\boldsymbol{\alpha}=\mathbf{2 . 5}$ & $\boldsymbol{\alpha}=\mathbf{3}$ & $\boldsymbol{\alpha}=\mathbf{3 . 5}$ & $\boldsymbol{\alpha}=\mathbf{4}$ & $\boldsymbol{\alpha}=\mathbf{4 . 5}$ & $\boldsymbol{\alpha}=\mathbf{5}$ \\
\hline$r=1$ & 0.38730 & 0.29360 & 0.23650 & 0.19810 & 0.17050 & 0.14960 \\
$r=2$ & 0.06800 & 0.05510 & 0.04640 & 0.04000 & 0.03520 & 0.03140 \\
$r=3$ & 0.01830 & 0.01510 & 0.01280 & 0.01110 & 0.00990 & 0.00880 \\
$r=4$ & 0.00550 & 0.00460 & 0.00390 & 0.00340 & 0.00300 & 0.00270 \\
$r=5$ & 0.00180 & 0.00150 & 0.00120 & 0.00110 & 0.00097 & 0.00087 \\
\hline
\end{tabular}


Table 4 Variance of lower records from inverted Kumaraswamy distribution for

$\alpha=2.5: 0.5: 5, \beta=0.5,(m \rightarrow-1, k=1)$.

\begin{tabular}{ccccccc}
\hline & $\boldsymbol{\alpha}=\mathbf{2 . 5}$ & $\boldsymbol{\alpha}=\mathbf{3}$ & $\boldsymbol{\alpha}=\mathbf{3 . 5}$ & $\boldsymbol{\alpha}=\mathbf{4}$ & $\boldsymbol{\alpha}=\mathbf{4 . 5}$ & $\boldsymbol{\alpha}=\mathbf{5}$ \\
\hline$r=1$ & 1.20980 & 0.43000 & 0.22167 & 0.13526 & 0.09113 & 0.06562 \\
$r=2$ & 0.02448 & 0.01486 & 0.00995 & 0.00720 & 0.00536 & 0.00421 \\
$r=3$ & 0.00267 & 0.00177 & 0.00124 & 0.00088 & 0.00071 & 0.00056 \\
$r=4$ & 0.00041 & 0.00027 & 0.00020 & 0.00015 & 0.00011 & 0.00009 \\
$r=5$ & 0.00007 & 0.00005 & 0.00003 & 0.00003 & 0.00002 & 0.00002 \\
\hline
\end{tabular}

Table 5 Mean of $d g o s$ from inverted Kumaraswamy distribution for $\alpha=5, \beta=0.5,(m=1, k=2)$.

\begin{tabular}{ccccccccc}
\hline $\boldsymbol{n}$ & $\boldsymbol{r}=\mathbf{1}$ & $\boldsymbol{r}=\mathbf{2}$ & $\boldsymbol{r}=\mathbf{3}$ & $\boldsymbol{r}=\mathbf{4}$ & $\boldsymbol{r}=\mathbf{5}$ & $\boldsymbol{r}=\mathbf{6}$ & $\boldsymbol{r}=\mathbf{7}$ & $\boldsymbol{r}=\mathbf{8}$ \\
\hline 1 & 0.25 & & & & & & & \\
2 & 0.3889 & 0.1111 & & & & & & \\
3 & 0.4881 & 0.1905 & 0.0714 & & & & & \\
4 & 0.5664 & 0.2531 & 0.1278 & 0.0526 & & & & \\
5 & 0.6317 & 0.3053 & 0.1748 & 0.0965 & 0.0417 & & & \\
6 & 0.6879 & 0.3504 & 0.2153 & 0.1343 & 0.0776 & 0.0345 & & \\
7 & 0.7376 & 0.3901 & 0.2511 & 0.1677 & 0.1093 & 0.0649 & 0.0294 & \\
8 & 0.7821 & 0.4257 & 0.2831 & 0.1976 & 0.1377 & 0.0922 & 0.0558 & 0.0256 \\
\hline
\end{tabular}

Table 6 Variance of dgos from inverted Kumaraswamy distribution for $\alpha=5, \beta=0.5,(m=1, k=2)$.

\begin{tabular}{ccccccccc}
\hline $\boldsymbol{n}$ & $\boldsymbol{r}=\mathbf{1}$ & $\boldsymbol{r}=\mathbf{2}$ & $\boldsymbol{r}=\mathbf{3}$ & $\boldsymbol{r}=\mathbf{4}$ & $\boldsymbol{r}=\mathbf{5}$ & $\boldsymbol{r}=\mathbf{6}$ & $\boldsymbol{r}=\mathbf{7}$ & $\boldsymbol{r}=\mathbf{8}$ \\
\hline 1 & 0.10420 & & & & & & & \\
2 & 0.15436 & 0.01546 & & & & & & \\
3 & 0.18946 & 0.02511 & 0.00590 & & & & & \\
4 & 0.21729 & 0.03224 & 0.01007 & 0.00303 & & & & \\
5 & 0.24076 & 0.03799 & 0.01334 & 0.00539 & 0.00186 & & & \\
6 & 0.26149 & 0.04282 & 0.01605 & 0.00736 & 0.00338 & 0.00131 & & \\
7 & 0.27985 & 0.04712 & 0.01835 & 0.00898 & 0.00465 & 0.00229 & 0.00094 & \\
8 & 0.29672 & 0.05098 & 0.02045 & 0.01035 & 0.00574 & 0.00320 & 0.00169 & 0.00064 \\
\hline
\end{tabular}

\subsection{Single Moment}

Theorem 2.1. Let $X_{1}, X_{2}, \ldots, X_{n}$ be $n$ continuous iid non negative random variables and follow inverted Kumaraswamy distribution given in (1.12), then the single moment of $r$ th dgos $(1 \leq r \leq n)$ is given by

$$
\begin{aligned}
\mu_{r, n, \tilde{m}, k}^{j} & =E\left(X_{d}^{j}(r, n, \tilde{m}, k)\right) \\
& =\beta c_{r-1} \sum_{t=1}^{r} \sum_{i=0}^{j}(-1)^{i}\left(\begin{array}{l}
j \\
i
\end{array}\right) a_{t}(r) \mathcal{B}\left(1-\frac{j-i}{\alpha}, \beta \gamma_{t}\right), \quad j-i<\alpha
\end{aligned}
$$

and in case of lower records, the single moment is given by

$$
\mu_{r, n,-1, k}^{j}=\frac{\beta^{r}}{(r-1) !} \sum_{i=0}^{j}\left(\begin{array}{c}
j \\
i
\end{array}\right)(-1)^{i+j+r-1} \frac{\partial^{r-1}}{\partial \beta^{r-1}} \mathcal{B}(1-i / \alpha, \beta), \quad i<\alpha
$$

where $j \in \mathbb{N}$ and $\mathcal{B}(a, b)=\int_{0}^{1} x^{a-1}(1-x)^{b-1}$ is a complete beta function.

Proof. In case of $d g o s$, for the inverted Kumaraswamy distribution given in (1.12), the single moment of $r^{\text {th }} d g o s$ is given by

$$
\mu_{r, n, \tilde{m}, k}^{j}=\alpha \beta c_{r-1} \sum_{t=1}^{r} a_{t}(r) \int_{0}^{\infty} x^{j}\left[1-(1+x)^{-\alpha}\right]^{\beta \gamma_{t}-1}(1+x)^{-\alpha-1} \mathrm{~d} x
$$

Putting $(1+x)^{-\alpha}=t$ and simplifying accordingly, we get

$$
\begin{aligned}
\mu_{r, n, \tilde{m}, k}^{j} & =\beta c_{r-1} \sum_{t=1}^{r} a_{t}(r) \int_{0}^{1}\left(t^{-1 / \alpha}-1\right)^{j}(1-t)^{\beta \gamma_{t}-1} \mathrm{~d} t \\
& =\beta c_{r-1} \sum_{t=1}^{r} \sum_{i=0}^{j} a_{t}(r)\left(\begin{array}{c}
j \\
i
\end{array}\right)(-1)^{i} \int_{0}^{1} t^{-(j-i) / \alpha}(1-t)^{\beta \gamma_{t}-1} \mathrm{~d} t
\end{aligned}
$$


Now, using the relation $\int_{0}^{1} x^{a-1}(1-x)^{b-1}=\mathcal{B}(a, b)$, we obtained the result.

In case of lower records, the $r^{\text {th }}$ single moment from inverted Kumaraswamy distribution is given by

$$
\mu_{r, n,-1, k}^{j}=\frac{\alpha \beta}{(r-1) !} \int_{0}^{\infty} x^{j}\left[-\ln \left(1-(1+x)^{-\alpha}\right)^{\beta}\right]^{r-1}(1+x)^{-\alpha-1}\left[1-(1+x)^{-\alpha}\right]^{\beta-1} d x
$$

Assuming $(1+x)^{-\alpha}=u$ and simplifying appropriately, we get

$$
\begin{aligned}
\mu_{r, n,-1, k}^{j} & =\frac{\beta^{r}}{(r-1) !} \int_{0}^{1}\left(u^{-1 / \alpha}-1\right)^{j}[-\ln (1-u)]^{r-1}(1-u)^{\beta-1} d u \\
& =\frac{\beta^{r}}{(r-1) !} \sum_{i=0}^{j}\left(\begin{array}{l}
j \\
i
\end{array}\right)(-1)^{i+j+r-1} \int_{0}^{1} u^{(1-i / \alpha)-1}(1-u)^{\beta-1}[\ln (1-u)]^{r-1} d u
\end{aligned}
$$

Using the relation $\int_{0}^{a} x^{\alpha-1}(a-x)^{\beta-1}\left[\ln ^{r}(a-x)\right] d x=a^{\alpha-1} \frac{\partial^{r}}{\partial \beta^{r}}\left[a^{\beta} \mathcal{B}(\alpha, \beta)\right]$, (Prudnikov et al. [32], p. 502), we obtained the result.

It is pertinent to mention that the computation of $\frac{\partial^{r}}{\partial \beta^{r}}\left[a^{\beta} \mathcal{B}(\alpha, \beta)\right]$ can further be simplified by using the recurrence relation

$$
\frac{\partial^{r} \mathcal{B}(\alpha, \beta)}{\partial \beta^{r}}=\sum_{k=0}^{r-1}\left[\psi^{(r-k-1)}(\beta)-\psi^{(r-k-1)}(\alpha+\beta)\right] \frac{\partial^{k} \mathcal{B}(\alpha, \beta)}{\partial \beta^{k}}
$$

where $\psi^{(r-k-1)}(x)$ is the $k$ th derivative of diagamma function given by $\psi(x)=\frac{d \log \Gamma(x)}{d x}=\frac{\Gamma(x)}{\Gamma(x)}, x>0$ and $\Gamma($.$) is the gamma function (\mathrm{Khan}$ et al. [33]).

Corollary 2.1. for $m_{1}=m_{2}=\ldots, m_{n-1}=m \neq-1$, the single moment of inverted Kumaraswamy distribution is given by

$$
\mu_{r, n, m, k}^{j}=\frac{\beta c_{r-1}}{(m+1)^{r-1}(r-1) !} \sum_{t=0}^{r-1} \sum_{i=1}^{j}(-1)^{i+t}\left(\begin{array}{c}
r-1 \\
t
\end{array}\right)\left(\begin{array}{l}
j \\
i
\end{array}\right) \mathcal{B}\left(1-\frac{j-i}{\alpha}, \beta \gamma_{r-t}\right), \underbrace{}_{j-i<\alpha}
$$

Remark 2.1. When $m=0, k=1$, the single moment of order statistics from inverted Kumaraswamy distribution is given by

$$
\mu_{n-r+1, n, 0,1}^{j}=\frac{\beta n !}{(n-r) !(r-1) !} \sum_{t=0}^{n-r} \sum_{i=0}^{j}(-1)^{i+t}\left(\begin{array}{c}
n-r \\
t
\end{array}\right)\left(\begin{array}{c}
j \\
i
\end{array}\right) \mathcal{B}\left(1-\frac{j-i}{\alpha}, \beta(n-r+t+1)\right) \underbrace{}_{j-i<\alpha}
$$

\subsection{Product Moments}

In this section, the product moment of $r^{\text {th }}$ and $s^{\text {th }}$ dgos is computed in terms of hypergeometric function from inverted Kumaraswamy distribution. Further, by choosing the different combination of the parameters of dgos (i.e., $m$ and $k$ ), we reduced the obtained result in terms of order statistics and lower record values. The obtained result is summarized in the form of theorem which is given below.

Theorem 2.2. Let $X_{1}, X_{2}, \ldots, X_{n}$ be $n$ continuous iid non negative random variables that follow inverted kumaraswamy distribution given in (1.12) and $X_{d}(i, n, \tilde{m}, k), i=1,2, \ldots, n$ be their corresponding dgos. For any $\lambda, l \in \mathbb{N}$ and $\gamma_{1: r}=\min \left(\gamma_{1}, \gamma_{2}, \ldots, \gamma_{r}\right)>\gamma_{r+1}, \ldots, \gamma_{s}$, the product moment of $r^{\text {th }}$ and $s^{\text {th }}$ dgos $(1 \leq r<s \leq n)$ is given by

$$
\begin{aligned}
\mu_{r, s, n, \tilde{m}, k}^{\lambda, l}= & E\left[X_{d}^{\lambda}(r, n, \tilde{m}, k) X_{d}^{l}(s, n, \tilde{m}, k)\right] \\
= & \beta^{2} c_{s-1} \sum_{i=1}^{r} \sum_{j=r+1}^{s} \sum_{p=0}^{\lambda} \sum_{q=0}^{l} a_{i}(r) a_{j}^{(r)}(s)\left(\begin{array}{c}
\lambda \\
p
\end{array}\right)\left(\begin{array}{c}
l \\
q
\end{array}\right) \frac{(-1)^{p+q}}{1-\left(\frac{\lambda-p}{\alpha}\right)} \\
& \times \mathcal{B}\left(\frac{l-\lambda+p-q}{\alpha}+2, \beta \gamma_{j}\right)_{3} F_{2}\left(1-\frac{\lambda-p}{\alpha}, 1-\beta\left(\gamma_{i}-\gamma_{j}\right), \frac{l-\lambda+p-q}{\alpha}+2 ; 2-\frac{\lambda-p}{\alpha}, \frac{l-\lambda+p-q}{\alpha}+\beta \gamma_{j}+2 ; 1\right)
\end{aligned}
$$


where $\lambda, l \in \mathbb{N}$ and ${ }_{3} F_{2}\left(a_{1}, a_{2}, a_{3} ; b_{1}, b_{2} ; x\right)$ is generalized hypergeometric function defined as follows:

$$
{ }_{3} F_{2}\left(a_{1}, a_{2}, a_{3} ; b_{1}, b_{2} ; x\right)=\sum_{t=0}^{\infty} \frac{\left(a_{1}\right)_{t}\left(a_{2}\right)_{t}\left(a_{3}\right)_{t}}{\left(b_{1}\right)_{t}\left(b_{2}\right)_{t}} \frac{x^{t}}{t !}
$$

(Mathai and Saxena [34]).

Proof. In view of (1.7), for the inverted Kumaraswamy distribution given in (1.12) the product moment for $s^{\text {th }}$ and $r^{\text {th }} d g o s$ is given by

$$
\begin{array}{r}
\mu_{r, s, n, \tilde{m}, k}^{\lambda, l}=c_{s-1} \sum_{i=1}^{r} \sum_{j=r+1}^{s} a_{i}(r) a_{j}^{(r)}(s) \int_{0}^{\infty} \int_{y}^{\infty} x^{\lambda} y^{l}[F(y)]^{\gamma_{j}}[F(x)]^{\gamma_{i}-\gamma_{j}} \frac{f(x)}{F(x)} \frac{f(y)}{F(y)} d x d y \\
0<y<x<\infty
\end{array}
$$

Consider

$$
\begin{aligned}
I(y) & =\int_{y}^{\infty} x^{\lambda}[F(x)]^{\gamma_{i}-\gamma_{j}} \frac{f(x)}{F(x)} d x \\
& =\alpha \beta \int_{y}^{\infty} x^{\lambda}\left[1-(1+x)^{-\alpha}\right]^{\beta\left(\gamma_{i}-\gamma_{j}\right)-1}(1+x)^{-\alpha-1} d x
\end{aligned}
$$

Let $(1+x)^{-\alpha}=t$ and simplifying accordingly, we have

$$
\begin{aligned}
& I(y)=\beta \int_{0}^{(1+y)^{-\alpha}}\left(t^{-1 / \alpha}-1\right)^{k}[1-t]^{\beta\left(\gamma_{i}-\gamma_{j}\right)-1} d t \\
= & \beta \sum_{p=0}^{\lambda}\left(\begin{array}{l}
\lambda \\
p
\end{array}\right)(-1)^{p} \int_{0}^{(1+y)^{-\alpha}} t^{1-(\lambda-p) / \alpha-1}[1-t]^{\beta\left(\gamma_{i}-\gamma_{j}\right)-1} d t \\
= & \beta \sum_{p=0}^{\lambda}\left(\begin{array}{l}
\lambda \\
p
\end{array}\right)(-1)^{p} \mathcal{B}_{(1+y)^{-\alpha}}\left(1-\frac{(\lambda-p)}{\alpha}, \beta\left(\gamma_{i}-\gamma_{j}\right)\right) \\
= & \beta \sum_{p=0}^{\lambda}\left(\begin{array}{l}
\lambda \\
p
\end{array}\right)(-1)^{p} \int_{0}^{(1+y)^{-\alpha}} t^{1-(\lambda-p) / \alpha-1}[1-t]^{\beta\left(\gamma_{i}-\gamma_{j}\right)-1} d t
\end{aligned}
$$

Therefore,

$$
\begin{aligned}
\mu_{r, s, n, \tilde{m}, k}^{\lambda, l}= & \alpha \beta^{2} c_{s-1} \sum_{i=1}^{r} \sum_{j=r+1}^{s} \sum_{p=0}^{\lambda} a_{i}(r) a_{j}^{(r)}(s)\left(\begin{array}{c}
\lambda \\
p
\end{array}\right) \frac{(-1)^{p}}{1-\left(\frac{\lambda-p}{\alpha}\right)} \\
& \times \int_{0}^{\infty} y^{l}\left[(1+y)^{-\alpha}\right]^{1-\left(\frac{\lambda-p}{\alpha}\right)}\left[1-(1+y)^{-\alpha}\right]^{\beta \gamma_{j}-1}(1+y)^{-\alpha-1} \\
& \times{ }_{2} F_{1}\left(1-\left(\frac{\lambda-p}{\alpha}\right), 1-\beta\left(\gamma_{i}-\gamma_{j}\right) ; 2-\left(\frac{\lambda-p}{\alpha}\right) ;(1+y)^{-\alpha}\right) d y
\end{aligned}
$$

Again putting $(1+y)^{-\alpha}=z$, the product moment of inverted Kumaraswamy distribution based on $d g o s$ is given by

$$
\begin{aligned}
\mu_{r, s, n, \tilde{m}, k}^{\lambda, l}= & \beta^{2} c_{s-1} \sum_{i=1}^{r} \sum_{j=r+1}^{s} \sum_{p=0}^{\lambda} a_{i}(r) a_{j}^{(r)}(s)\left(\begin{array}{l}
\lambda \\
p
\end{array}\right) \frac{(-1)^{p}}{1-\left(\frac{\lambda-p}{\alpha}\right)} \int_{0}^{1}\left(z^{-1 / \alpha}-1\right)^{l} z^{1-\left(\frac{\lambda-p}{\alpha}\right)} \\
& \times(1-z)_{2}^{\beta \gamma_{j}-1} F_{1}\left(1-\left(\frac{\lambda-p}{\alpha}\right), 1-\beta\left(\gamma_{i}-\gamma_{j}\right) ; 2-\left(\frac{\lambda-p}{\alpha}\right) ; z\right) d z \\
= & \beta^{2} c_{s-1} \sum_{i=1}^{r} \sum_{j=r+1}^{s} \sum_{p=0}^{\lambda} \sum_{q=0}^{l}\left(\begin{array}{c}
\lambda \\
p
\end{array}\right)\left(\begin{array}{c}
l \\
q
\end{array}\right) a_{i}(r) a_{j}^{(r)}(s) \frac{(-1)^{p+q}}{1-\left(\frac{\lambda-p}{\alpha}\right)} \int_{0}^{1} z^{(l-q) / \alpha} z^{1-\left(\frac{\lambda-p}{\alpha}\right)} \\
& \times(1-z)_{2}^{\beta \gamma_{j}-1} F_{1}\left(1-\left(\frac{\lambda-p}{\alpha}\right), 1-\beta\left(\gamma_{i}-\gamma_{j}\right) ; 2-\left(\frac{\lambda-p}{\alpha}\right) ; z\right) d z
\end{aligned}
$$


or

$$
\begin{aligned}
\mu_{r, s, n, \tilde{m}, k}^{k \lambda, l}= & \beta^{2} c_{s-1} \sum_{i=1}^{r} \sum_{j=r+1}^{s} \sum_{p=0}^{\lambda} \sum_{q=0}^{l}\left(\begin{array}{c}
\lambda \\
p
\end{array}\right)\left(\begin{array}{l}
l \\
q
\end{array}\right) a_{i}(r) a_{j}^{(r)}(s) \frac{(-1)^{p+q}}{1-\left(\frac{\lambda-p}{\alpha}\right)} \int_{0}^{1} z^{1+(l-q-k+p) / \alpha} \\
& \times(1-z)_{2}^{\beta \gamma_{j}-1} F_{1}\left(1-\left(\frac{\lambda-p}{\alpha}\right), 1-\beta\left(\gamma_{i}-\gamma_{j}\right) ; 2-\left(\frac{\lambda-p}{\alpha}\right) ; z\right) d z
\end{aligned}
$$

Now using the relation (Mathai and Saxena [34])

$$
\int_{0}^{1} u^{a-1}(1-u)_{2}^{b-1} F_{1}(c, d ; e ; u) d u=\mathcal{B}(a, b)_{3} F_{2}(c, d, a ; e ; a+b ; 1)
$$

we have,

$$
\begin{aligned}
\mu_{r, s, n, \tilde{m}, k}^{\lambda, l}= & \beta^{2} c_{s-1} \sum_{i=1}^{r} \sum_{j=r+1}^{s} \sum_{p=0}^{\lambda} \sum_{q=0}^{l} a_{i}(r) a_{j}^{(r)}(s)\left(\begin{array}{c}
\lambda \\
p
\end{array}\right)\left(\begin{array}{c}
l \\
q
\end{array}\right) \frac{(-1)^{p+q}}{1-\left(\frac{\lambda-p}{\alpha}\right)} \\
& \times \mathcal{B}\left(\frac{l-\lambda+p-q}{\alpha}+2, \beta \gamma_{j}\right) \quad 3 F_{2}\left(1-\frac{\lambda-p}{\alpha}, 1-\beta\left(\gamma_{i}-\gamma_{j}\right), \frac{l-\lambda+p-q}{\alpha}+2 ; 2-\frac{\lambda-p}{\alpha}, \frac{l-\lambda+p-q}{\alpha}+\beta \gamma_{j}+2 ; 1\right)
\end{aligned}
$$

Hence, we get the result.

However, in case of lower records, an exact expression for product moment from inverted Kumaraswamy distribution could not be obtained.

Corollary 2.2. For $m_{1}=m_{2}, \ldots, m_{n-1}=m \neq-1$, the product moment from inverted Kumaraswamy distribution is given as

$$
\begin{aligned}
\mu_{r, s, n, m, k}^{\lambda, l}= & \frac{\beta^{2} c_{s-1}}{(m+1)^{s-2}(r-1) !(s-r-1) !} \sum_{i=0}^{r-1} \sum_{j=0}^{s-r-1} \sum_{p=0}^{\lambda} \sum_{q=0}^{l} \frac{(-1)^{p+q+i+j}}{1-\left(\frac{\lambda-p}{\alpha}\right)} \\
& \times\left(\begin{array}{c}
\lambda \\
p
\end{array}\right)\left(\begin{array}{c}
l \\
q
\end{array}\right)\left(\begin{array}{c}
r-1 \\
i
\end{array}\right)\left(\begin{array}{c}
s-r-1 \\
j
\end{array}\right) \mathcal{B}\left(\frac{l-\lambda+p-q}{\alpha}+2, \beta \gamma_{s-j}\right) \\
& \times{ }_{3} F_{2}\left(1-\frac{\lambda-p}{\alpha}, 1-\beta\left(\gamma_{r-i}-\gamma_{s-j}\right), \frac{l-\lambda+p-q}{\alpha}+2 ; 2-\frac{\lambda-p}{\alpha}, \frac{l-\lambda+p-q}{\alpha}+\beta \gamma_{s-j}+2 ; 1\right)
\end{aligned}
$$

Remark 2.2. In case of order statistics, i.e., $m=0, k=1$, the product moment from inverted Kumaraswamy distribution is given by

$$
\begin{aligned}
\mu_{n-r+1, n-s+1, n, 0,1}^{\lambda, l} & =\frac{\beta^{2} n !}{(n-s) !(r-1) !(s-r-1) !} \sum_{i=0}^{n-r} \sum_{j=0}^{s-r-1} \sum_{p=0}^{\lambda} \sum_{q=0}^{l} \frac{(-1)^{i+j+p+q}}{1-\left(\frac{\lambda-p}{\alpha}\right)}\left(\begin{array}{c}
n-r \\
i
\end{array}\right) \\
& \times\left(\begin{array}{c}
s-r-1 \\
j
\end{array}\right)\left(\begin{array}{c}
\lambda \\
p
\end{array}\right)\left(\begin{array}{l}
l \\
q
\end{array}\right) \mathcal{B}\left(\frac{l-\lambda+p-q}{\alpha}+2, \beta(n-s+j+1)\right) \\
& { }_{3} F_{2}\left(1-\frac{\lambda-p}{\alpha}, 1-\beta(s-r-j+i), \frac{l-\lambda+p-q}{\alpha}+2 ; 2-\frac{\lambda-p}{\alpha}, \frac{l-\lambda+p-q}{\alpha}+\beta(n-s+j+1)+2 ; 1\right)
\end{aligned}
$$

\subsection{Conditional Moments}

In this section, we have obtained the conditional moments of $s^{\text {th }}$ given $r^{\text {th }} d g o s\left(X_{d(s, n, \tilde{m}, k)} \mid X_{d(r, n, \tilde{m}, k)}\right)$ and $r^{\text {th }}$ given $s^{\text {th }} d g o s\left(X_{d(r, n, \tilde{m}, k)} \mid X_{d(s, n, \tilde{m}, k)}\right)$ from inverted Kumaraswamy Distribution in terms of incomplete beta function.

Theorem 2.3. The $q^{\text {th }}(q \in \mathbb{N})$ conditional moment of $s^{\text {th }}$ given $r^{\text {th }}$ dgos $\left(X_{d(s, n, \tilde{m}, k)} \mid X_{d(r, n, \tilde{m}, k)}=x\right), 1 \leq r<s \leq n$, from inverted Kumaraswamy distribution is given by

$$
\begin{aligned}
\mu_{X_{d(s, n, \tilde{m}, k)}^{q} \mid X_{d(r, n, \tilde{m}, k)}}^{q}(y \mid x)= & \beta \frac{c_{s-1}}{c_{r-1}} \sum_{j=r+1}^{s} a_{j}^{(r)}(s) \sum_{i=0}^{q}\left(\begin{array}{c}
q \\
i
\end{array}\right)(-1)^{i+1}\left[1-(1+x)^{-\alpha}\right]^{-\beta \gamma_{j}} \\
& \times \mathcal{B}_{1-(1+x)^{-\alpha}}\left(\beta \gamma_{j}, 1+\frac{(i-q)}{\alpha}\right), \quad 0<y<x<\infty
\end{aligned}
$$


where

$$
\mathcal{B}_{v}(a, b)=\int_{0}^{v} x^{a-1}(1-x)^{b-1} d x
$$

is an upper incomplete beta function.

Proof. The conditional moment of $X_{d(s, n, \tilde{m}, k)} \mid X_{d(r, n, \tilde{m}, k)}=x$ is given by

$$
\mu_{X_{d(s, n, \tilde{m}, k)}^{q} \mid X_{d(r, n, \tilde{m}, k)}}^{q}(y \mid x)=\int_{0}^{x} y^{q} f_{s \mid r}(y \mid x) d y, \quad 0<y<x<\infty
$$

From (1.4), (1.12) and (1.13), we have

$$
\begin{aligned}
\mu_{X_{d(s, n, \tilde{m}, k)}^{q} \mid X_{d(r, n, n, k, k)}}(y \mid x)= & \alpha \beta \frac{c_{s-1}}{c_{r-1}} \sum_{j=r+1}^{s} a_{j}^{(r)}(s) \int_{0}^{x} y^{q}\left[\frac{1-(1+y)^{-\alpha}}{1-(1+x)^{-\alpha}}\right]^{\beta \gamma_{j}} \\
& \times \frac{(1+y)^{-\alpha-1}\left[1-(1+y)^{-\alpha}\right]^{\beta-1}}{\left[1-(1+y)^{-\alpha}\right]^{\beta}} d y \\
= & \alpha \beta \frac{c_{s-1}}{c_{r-1}} \sum_{j=r+1}^{s} a_{j}^{(r)}(s)\left[1-(1+x)^{-\alpha}\right]^{-\beta \gamma_{j}} \\
& \times \int_{0}^{x} y^{q}\left[1-(1+y)^{-\alpha}\right]^{\beta \gamma_{j}+\beta-1-\beta}(1+y)^{-\alpha-1} d y .
\end{aligned}
$$

Putting $(1+y)^{-\alpha}=t$, we get

$$
\begin{aligned}
\mu_{X_{d(s, n, \tilde{m}, k)}^{q} \mid X_{d(r, n, \tilde{m}, k)}(y \mid x)=} & \beta \frac{c_{s-1}}{c_{r-1}} \sum_{j=r+1}^{s} a_{j}^{(r)}(s)\left[1-(1+x)^{-\alpha}\right]^{-\beta \gamma_{j}} \\
& \times \int_{(1+x)^{-\alpha}}^{1}\left(t^{-1 / \alpha}-1\right)^{q}(1-t)^{\beta \gamma_{j}-1} d t \\
= & \beta \frac{c_{s-1}}{c_{r-1}} \sum_{j=r+1}^{s} \sum_{l=0}^{q} a_{j}^{(r)}(s)\left(\begin{array}{l}
q \\
l
\end{array}\right)(-1)^{l}\left[1-(1+x)^{-\alpha}\right]^{-\beta \gamma_{j}} \\
& \times \int_{(1+x)^{-\alpha}}^{1}\left(t^{-1 / \alpha}\right)^{q-l}(1-t)^{\beta \gamma_{j}-1} d t
\end{aligned}
$$

Setting $(1-t)=u$ and then using the relation given in (2.9), we obtained the result.

Theorem 2.4. The $q^{\text {th }}(q \in \mathbb{N})$, conditional moment of $X_{d(r, n, \tilde{m}, k)} \mid X_{d(s, n, \tilde{m}, k)}=y, 1 \leq r<s \leq n$, from inverted Kumaraswamy distribution is given by

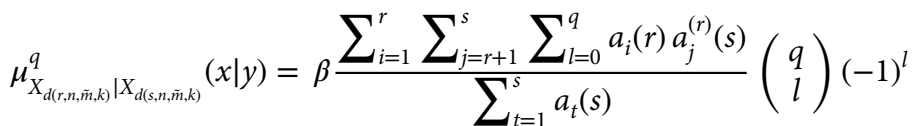

$$
\begin{aligned}
& \times\left[1-(1+y)^{-\alpha}\right]^{\beta\left(\gamma_{j}-\gamma_{t}\right)} \mathcal{B}_{(1+y)^{-\alpha}}\left(\frac{(l-q)}{\alpha}+1, \quad \beta\left(\gamma_{i}-\gamma_{j}\right)\right)
\end{aligned}
$$

for all $\gamma_{1: r}=\min \left(\gamma_{1}, \gamma_{2}, \ldots, \gamma_{r}\right)>\gamma_{r+1}, \ldots, \gamma_{s}$.

Proof. The conditional moment of $X_{d(r, n, \tilde{m}, k)} \mid X_{d(s, n, \tilde{m}, k)}=y$, is given by

$$
\mu_{X_{d(r, n, \tilde{m}, k), k}^{q} \mid X_{d(s, n, \tilde{m}, k)}}^{q}(x \mid y)=\int_{\infty}^{y} x^{q} f_{r \mid s}(x \mid y) d x, \quad 0<y<x<\infty
$$


In view of (1.3), (1.12) and (1.13), we have

$$
\begin{aligned}
\mu_{X_{d(r, n, \tilde{m}, k)}^{q} \mid X_{d(s, n, \tilde{m}, k)}(x \mid y)} & =\alpha \beta \frac{\sum_{i=1}^{r} a_{i}(r) \sum_{j=r+1}^{s} a_{j}^{(r)}(s)}{\sum_{t=1}^{s} a_{t}(s)}\left[1-(1+y)^{-\alpha}\right]^{\beta\left(\gamma_{j}-\gamma_{t}\right)} \\
& \times \int_{y}^{\infty} x^{q}\left[1-(1+x)^{-\alpha}\right]^{\beta\left(\gamma_{i}-\gamma_{j}-1\right)}(1+x)^{-\alpha-1}\left[1-(1+x)^{-\alpha}\right]^{\beta-1} d x
\end{aligned}
$$

Putting $(1+x)^{-\alpha}=z$ and simplifying accordingly, we get

$$
\begin{aligned}
& \mu_{X_{d(r, n, \tilde{m}, k)}^{q} \mid X_{d(s, n, \tilde{m}, k)}(x \mid y)}=\beta \frac{\sum_{i=1}^{r} \sum_{j=r+1}^{s} a_{i}(r) a_{j}^{(r)}(s)}{\sum_{t=1}^{s} a_{t}(s)}\left[1-(1+y)^{-\alpha}\right]^{\beta\left(\gamma_{j}-\gamma_{t}\right)} \\
& \times \int_{0}^{(1+y)^{-\alpha}}\left(z^{-1 / \alpha}-1\right)^{q}(1-z)^{\beta\left(\gamma_{i}-\gamma_{j}\right)-1} d z \\
&=\beta \frac{\sum_{i=1}^{r} \sum_{j=r+1}^{s} a_{i}(r) a_{j}^{(r)}(s)}{\sum_{t=1}^{s} a_{t}(s)}\left[1-(1+y)^{-\alpha}\right]^{\beta\left(\gamma_{j}-\gamma_{t}\right)} \\
& \times \int_{0}^{(1+y)^{-\alpha}} z^{-q / \alpha}\left(1-z^{1 / \alpha}\right)^{q}(1-z)^{\beta\left(\gamma_{i}-\gamma_{j}\right)-1} d z \\
&=\beta \frac{\sum_{i=1}^{r} \sum_{j=r+1}^{s} \sum_{p=0}^{q} a_{i}(r) a_{j}^{(r)}(s)}{\sum_{t=1}^{s} a_{t}(s)}\left(\begin{array}{l}
q \\
p
\end{array}\right)(-1)^{p} \\
& \times\left[1-(1+y)^{-\alpha}\right]^{\beta\left(\gamma_{j}-\gamma_{t}\right)} \int_{0}^{(1+y)^{-\alpha}} z^{(p-q) / \alpha}(1-z)^{\beta\left(\gamma_{i}-\gamma_{j}\right)-1} d z
\end{aligned}
$$

Using (2.9), we obtained the result.

\section{MLE FOR THE SHAPE PARAMETERS $\alpha$ AND $\beta$}

In this section, we have deduced expressions for MLE of the shape parameters of the inverted Kumaraswamy distribution based on $d g o s$.

Estimation of parameters through MLE based on gos and dgos has been discussed by many authors. Habibullah and Ahsanullah [35] have discussed the parameters of Pareto distribution based on gos. MLE and Bayes estimator of the parameters of Burr-XII distribution based on gos have been obtained by Jaheen [36] after that Malinowska et al. [37] estimated the location and scale parameter of Burr-XII distribution for gos. Bayesian and nonBayesian estimation for Weibull distribution based on gos have been given by Abo-eleneen [38]. Abo-Elfotouh and Nassar [39] have considered the MLE and Bayesion estimation of parameters of Weibull extension model for gos. Safi and Ahmed [27] have discussed the MLE for the parameter of Kumaraswamy distribution based on gos. MLE and Bayesian estimator for the parameter of Kumaraswamy distribution based on gos have been obtained by El-Deen et al. [28]. Kim [40] have discussed the parameter estimation of generalized exponential distribution under dgos. MLE, UMVUE, and Bayes estimates of stress-strength reliability based on gos for exponential distribution have been Khan and Khatoon [41].

In view of (1.1) and (1.12) the likelihood function for inverted Kumaraswamy distribution is given by

$$
\begin{aligned}
L(\alpha, \beta)= & k \alpha^{n} \beta^{n}\left(\prod_{j=1}^{n-1} \gamma_{j}\right)\left(\prod_{j=1}^{n}\left(1+x_{i}\right)^{-(\alpha+1)}\right)\left(\prod_{i=1}^{n-1}\left[1-\left(1+x_{i}\right)^{-\alpha}\right]^{\beta\left(m_{i}+1\right)-1}\right) \\
& \times\left[1-\left(1+x_{n}\right)^{-\alpha}\right]^{\beta k-1}, \quad 0<x_{n}<x_{n-1}<\ldots<x_{2}<x_{1}<\infty
\end{aligned}
$$

The log likelihood function of (3.1) is given by

$$
\begin{aligned}
l(\alpha, \beta)= & \ln k+n \ln \alpha+n \ln \beta+\sum_{i=1}^{n-1} \ln \gamma_{j}-(\alpha+1) \sum_{i=1}^{n} \ln \left(1+x_{i}\right) \\
& +\sum_{i=1}^{n-1}\left[\beta\left(m_{i}+1\right)-1\right] \ln \left[1-\left(1+x_{i}\right)^{-\alpha}\right]+(\beta k-1) \ln \left[1-\left(1+x_{n}\right)^{-\alpha}\right]
\end{aligned}
$$


Differentiating both sides of (3.2) w.r.t. $\alpha, \beta$ and equating to zero, we get

$$
\begin{aligned}
& \frac{n}{\alpha}-\sum_{i=1}^{n} \ln \left(1+x_{i}\right)-\sum_{i=1}^{n-1}\left[\beta\left(m_{i}+1\right)-1\right] \frac{\left(1+x_{i}\right)^{-\alpha} \ln \left(1+x_{i}\right)}{1-\left(1+x_{i}\right)^{-\alpha}} \\
& -(\beta k-1) \frac{\left(1+x_{n}\right)^{-\alpha} \ln \left(1+x_{n}\right)}{1-\left(1+x_{n}\right)^{-\alpha}}=0 \\
& \frac{n}{\beta}+\sum_{i=1}^{n-1}\left(m_{i}+1\right) \ln \left[1-\left(1+x_{i}\right)^{-\alpha}\right]+k \ln \left[1-\left(1+x_{n}\right)^{-\alpha}\right]=0
\end{aligned}
$$

In case of lower records $\left[\left(X_{L_{(1)}}, X_{L_{(2)}}, \ldots, X_{L_{(n)}}\right)=\left(x_{1}, x_{2}, \ldots, x_{n}\right)\right]$, the likelihood equation is given by

$$
L(\alpha, \beta)=\alpha^{n} \beta^{n}\left[1-\left(1+x_{n}\right)^{-\alpha}\right]^{\beta} \prod_{i=1}^{n} \frac{\left(1+x_{i}\right)^{-(\alpha+1)}}{1-\left(1+x_{i}\right)^{-\alpha}}
$$

And the MLE of $\alpha$ and $\beta$ in case of lower records can be obtained by solving the normal equations

$$
\frac{n}{\alpha}-\sum_{i=1}^{n} \ln \left(1+x_{i}\right)+\sum_{i=1}^{n} \frac{\left(1+x_{i}\right)^{-\alpha} \ln \left(1+x_{i}\right)}{1-\left(1+x_{i}\right)^{-\alpha}}-\beta \frac{\left(1+x_{n}\right)^{-\alpha} \ln \left(1+x_{n}\right)}{1-\left(1+x_{n}\right)^{-\alpha}}=0
$$

and

$$
\frac{n}{\beta}+\ln \left[1-\left(1+x_{n}\right)^{-\alpha}\right]=0
$$

From (3.3)-(3.7), the exact expression for MLE of $\alpha$ and $\beta$ cannot be obtained directly. Therefore, an iteration method (Newton-Raphson) is applied to obtain the MLE of $\alpha$ and $\beta$.

\section{UMVUE AND MLE OF $\beta$ WHEN $\alpha$ IS KNOWN}

In this section, we have obtained UMVUE and MLE of parameter $\beta$ of the inverted Kumaraswamy distribution based on $d g o s$ when $\alpha$ is known say $\alpha_{0}$.

Replacing $\alpha=\alpha_{0}$ in the equation (3.1) and re-write the joint $p d f$ of $n d g o s$ from inverted Kumaraswamy distribution as

$$
f\left(x_{1}, x_{2}, \ldots, x_{n}\right)=k \alpha^{n} \beta^{n}\left(\prod_{j=1}^{n-1} \gamma_{j}\right)\left(\prod_{i=1}^{n} \frac{\left(1+x_{i}\right)^{-(\alpha+1}}{\left[1-\left(1+x_{i}\right)^{-\alpha}\right]}\right) e^{-\beta \omega}
$$

where

$$
\omega=-\sum_{i=1}^{n-1}\left(m_{i}+1\right) \ln \left[1-\left(1+x_{i}\right)^{-\alpha_{0}}\right]-k \ln \left[1-\left(1+x_{n}\right)^{-\alpha_{0}}\right]
$$

From equation (4.1), it can be easily seen that $\omega$ is a complete sufficient statistics for $\beta$. From the normalized spacing of $d g o s$, one can easily prove that $\omega$ follows gamma distribution with shape parameter $n$ and scale parameter $1 / \beta$ denoting $G(n, 1 / \beta)$ (see Arshad and Jamal [20]). Therefore,

$$
E(\omega)=(n-1) / \beta
$$

and hence the UMVUE of $\beta$ is

$$
\hat{\beta}_{U M V U}=\frac{n-1}{\omega}
$$

Further, adopting the same procedure described in the previous section (3), the MLE of $\beta$ is given by

$$
\hat{\beta}_{M L}=\frac{n}{\omega}
$$


In case of lower records, UMVUE and MLE can be obtained as

$$
\hat{\beta}_{U M V U}=\frac{n-1}{-\ln \left(2 X_{L_{(n)}}-X_{L_{(n)}}^{2}\right)}
$$

and

$$
\hat{\beta}_{M L}=\frac{n}{-\ln \left(2 X_{L_{(n)}}-X_{L_{(n)}}^{2}\right)}
$$

where $X_{L_{(n)}}, n=1,2, \ldots$ denotes the lower record value of the sequence of the random variables. For more detail regarding this result, one can refer to Zghoul [42].

\section{SIMULATION STUDY}

In this section, the MLE for both the shape parameters, MLE and UMVUE of one shape parameter by taking another one is known of inverted Kumaraswamy distribution based on order statistics and lower records are computed. Here, R software is used for data simulation and computation purpose.

When both the shape parameters are unknown, we considered a Monte Carlo method of simulation to obtain the MLEs for the parameters by considering that $(\alpha, \beta)=((0.5,0.5),(0.5,1),(1,0.5),(1,1),(2.2,2.1))$, sample size $n=20,40, \ldots, 100$ for order statistics and for lower records $n=4,6, \ldots, 12$. For the entire simulation, we consider 1000 replication of the process and the average estimate, bias, and mean square error (MSE) are computed for order statistics and lower records which are reported in Tables 7 and 8 respectively.

For another case, i.e. , when one shape parameter $(\alpha)$ is known, the data is generated from $G(n, 1 / \beta)$ and the MLE and UMVUE are obtained for $\beta=0.5,1,2.5$ for fixed value of $\alpha, n=20,40, \ldots, 100$ for order statistics and $n=4,6, \ldots, 12$ for lower records. Based on 1000 repetition, the average MSE for MLE and UMVUE and average bias for MLE also are noted which are listed in Tables 9 and 10 for order statistics and lower records, respectively.

\begin{tabular}{|c|c|c|c|c|c|c|c|}
\hline \multirow[t]{2}{*}{$(\alpha, \beta)$} & \multirow[t]{2}{*}{$n$} & \multicolumn{3}{|c|}{$\alpha$} & \multicolumn{3}{|c|}{$\beta$} \\
\hline & & MLE & Bias & MSE & MLE & Bias & MSE \\
\hline \multirow[t]{5}{*}{$(0.5,0.5)$} & 20 & 0.59358 & 0.09358 & 0.06032 & 0.56703 & 0.06703 & 0.03495 \\
\hline & 40 & 0.54608 & 0.04608 & 0.02514 & 0.53022 & 0.03022 & 0.01283 \\
\hline & 60 & 0.52632 & 0.02632 & 0.01356 & 0.51934 & 0.01934 & 0.00714 \\
\hline & 80 & 0.51961 & 0.01961 & 0.00910 & 0.51365 & 0.01365 & 0.00526 \\
\hline & 100 & 0.51733 & 0.01733 & 0.00702 & 0.51260 & 0.01260 & 0.00437 \\
\hline \multirow[t]{5}{*}{$(0.5,1)$} & 20 & 0.57640 & 0.07640 & 0.04608 & 1.19448 & 0.19448 & 0.26841 \\
\hline & 40 & 0.53440 & 0.03440 & 0.01444 & 1.06839 & 0.06839 & 0.06219 \\
\hline & 60 & 0.51871 & 0.01871 & 0.00840 & 1.04760 & 0.04760 & 0.03773 \\
\hline & 80 & 0.51417 & 0.01417 & 0.00605 & 1.03265 & 0.03265 & 0.02480 \\
\hline & 100 & 0.50881 & 0.00881 & 0.00473 & 1.02427 & 0.02427 & 0.01987 \\
\hline \multirow[t]{5}{*}{$(1,0.5)$} & 20 & 1.16622 & 0.16622 & 0.24372 & 0.56160 & 0.06160 & 0.03262 \\
\hline & 40 & 1.08815 & 0.08815 & 0.09383 & 0.53022 & 0.03022 & 0.01304 \\
\hline & 60 & 1.04808 & 0.04808 & 0.05171 & 0.51787 & 0.01787 & 0.00768 \\
\hline & 80 & 1.04051 & 0.04051 & 0.03823 & 0.51435 & 0.01435 & 0.00535 \\
\hline & 100 & 1.02548 & 0.02548 & 0.03034 & 0.51053 & 0.01053 & 0.00371 \\
\hline \multirow[t]{5}{*}{$(1,1)$} & 20 & 1.12171 & 0.12171 & 0.13265 & 1.15090 & 0.15090 & 0.17176 \\
\hline & 40 & 1.05221 & 0.05221 & 0.05487 & 1.07467 & 0.07466 & 0.06503 \\
\hline & 60 & 1.04386 & 0.04386 & 0.03461 & 1.04669 & 0.04669 & 0.03704 \\
\hline & 80 & 1.03591 & 0.03591 & 0.02354 & 1.03709 & 0.03709 & 0.02622 \\
\hline & 100 & 1.03215 & 0.03215 & 0.02009 & 1.03069 & 0.03069 & 0.02180 \\
\hline \multirow[t]{5}{*}{$(2.5,2.5)$} & 20 & 2.75690 & 0.25690 & 0.54450 & 3.08986 & 0.58986 & 0.91808 \\
\hline & 40 & 2.61797 & 0.11797 & 0.21485 & 2.72915 & 0.22915 & 0.60108 \\
\hline & 60 & 2.57153 & 0.07153 & 0.14338 & 2.67798 & 0.17798 & 0.38361 \\
\hline & 80 & 2.57017 & 0.07017 & 0.10260 & 2.62625 & 0.12625 & 0.24075 \\
\hline & 100 & 2.54426 & 0.04426 & 0.07461 & 2.59075 & 0.09075 & 0.16908 \\
\hline
\end{tabular}

Table 7 MLE of parameters of inverted Kumaraswamy distribution based on order statistics. 
Table 8 MLE of parameters of inverted Kumaraswamy distribution based on lower records.

\begin{tabular}{|c|c|c|c|c|c|c|c|}
\hline \multirow[t]{2}{*}{$(\alpha, \beta)$} & \multirow[t]{2}{*}{$n$} & \multicolumn{3}{|c|}{$\alpha$} & \multicolumn{3}{|c|}{$\beta$} \\
\hline & & MLE & Bias & MSE & MLE & Bias & MSE \\
\hline \multirow[t]{5}{*}{$(0.5,0.5)$} & 4 & 0.95799 & 0.45799 & 1.10777 & 0.89634 & 0.39634 & 1.97488 \\
\hline & 6 & 0.83490 & 0.33490 & 0.64561 & 0.76025 & 0.26025 & 1.10315 \\
\hline & 8 & 0.80013 & 0.30013 & 0.50635 & 0.75618 & 0.25618 & 0.65685 \\
\hline & 10 & 0.75162 & 0.25162 & 0.33120 & 0.67128 & 0.17128 & 0.14820 \\
\hline & 12 & 0.68433 & 0.18433 & 0.18827 & 0.63725 & 0.13725 & 0.12061 \\
\hline \multirow[t]{5}{*}{$(0.5,1)$} & 4 & 0.88952 & 0.38952 & 0.63288 & 1.89668 & 0.89668 & 5.12380 \\
\hline & 6 & 0.78236 & 0.28236 & 0.36934 & 1.67542 & 0.67542 & 2.82767 \\
\hline & 8 & 0.71155 & 0.21155 & 0.20371 & 1.48849 & 0.48849 & 1.98405 \\
\hline & 10 & 0.65328 & 0.15328 & 0.13289 & 1.30778 & 0.30778 & 0.54395 \\
\hline & 12 & 0.61705 & 0.11705 & 0.08913 & 1.28827 & 0.28827 & 0.48011 \\
\hline \multirow[t]{5}{*}{$(1,0.5)$} & 4 & 1.79209 & 0.79209 & 2.96595 & 0.91618 & 0.41618 & 2.25161 \\
\hline & 6 & 1.56005 & 0.56005 & 1.36735 & 0.71638 & 0.21638 & 0.27241 \\
\hline & 8 & 1.50964 & 0.50964 & 0.99735 & 0.67093 & 0.17093 & 0.18000 \\
\hline & 10 & 1.31369 & 0.31369 & 0.65002 & 0.61446 & 0.11446 & 0.08548 \\
\hline & 12 & 1.29930 & 0.29930 & 0.49603 & 0.60035 & 0.10035 & 0.06708 \\
\hline \multirow[t]{5}{*}{$(1,1)$} & 4 & 1.58203 & 0.58203 & 1.45794 & 1.74879 & 0.74879 & 2.35062 \\
\hline & 6 & 1.40364 & 0.40364 & 0.74666 & 1.61671 & 0.61671 & 1.16099 \\
\hline & 8 & 1.32452 & 0.32452 & 0.53731 & 1.51269 & 0.51269 & 0.80420 \\
\hline & 10 & 1.22040 & 0.22039 & 0.27974 & 1.31340 & 0.31340 & 0.67306 \\
\hline & 12 & 1.19506 & 0.19506 & 0.25015 & 1.24222 & 0.24222 & 0.40805 \\
\hline \multirow[t]{5}{*}{$(2.5,2.5)$} & 4 & 3.00554 & 0.50554 & 1.62537 & 3.30193 & 0.80193 & 2.54828 \\
\hline & 6 & 2.95440 & 0.45440 & 1.33555 & 3.01969 & 0.51969 & 1.72553 \\
\hline & 8 & 2.88874 & 0.38874 & 0.97995 & 2.93177 & 0.43177 & 1.20719 \\
\hline & 10 & 2.81722 & 0.31722 & 0.71950 & 2.85483 & 0.35483 & 0.88320 \\
\hline & 12 & 2.77335 & 0.27335 & 0.58612 & 2.81584 & 0.31584 & 0.69187 \\
\hline
\end{tabular}

Table 9 MLE and UMVUE of $\beta$ when $\alpha$ is known for inverted Kumaraswamy distribution based on order statistics.

\begin{tabular}{ccccccc}
\hline $\boldsymbol{\beta}$ & $\boldsymbol{n}$ & $\hat{\boldsymbol{\beta}}_{\text {MLE }}$ & Bias $_{(\text {MLE })}$ & $\boldsymbol{M S E}_{(\text {MLE })}$ & $\hat{\boldsymbol{\beta}}_{\text {UMVUE }}$ & MSE $_{(\text {UMVUE })}$ \\
\hline$(\alpha=0.5)$ & & & & & & \\
0.5 & 20 & 0.52075 & 0.02075 & 0.01589 & 0.49471 & 0.01398 \\
& 40 & 0.50993 & 0.00993 & 0.00664 & 0.49718 & 0.00623 \\
& 60 & 0.50549 & 0.00549 & 0.00429 & 0.49706 & 0.00412 \\
& 80 & 0.50359 & 0.00359 & 0.00318 & 0.49729 & 0.00310 \\
& 100 & 0.50583 & 0.00583 & 0.00271 & 0.50077 & 0.00262 \\
1 & 20 & 1.06023 & 0.06023 & 0.06709 & 1.00722 & 0.05733 \\
& 40 & 1.03356 & 0.03356 & 0.02779 & 1.00772 & 0.02540 \\
& 60 & 1.01423 & 0.01423 & 0.01693 & 0.99732 & 0.01619 \\
& 80 & 1.01169 & 0.01169 & 0.01370 & 0.99904 & 0.01322 \\
& 100 & 1.01347 & 0.01347 & 0.01038 & 1.00333 & 0.01000 \\
2.5 & 20 & 2.59716 & 0.09716 & 0.36898 & 2.46730 & 0.32555 \\
& 40 & 2.55618 & 0.05618 & 0.16035 & 2.49227 & 0.14949 \\
& 60 & 2.53333 & 0.03333 & 0.11962 & 2.49111 & 0.11467 \\
& 80 & 2.53456 & 0.03456 & 0.08193 & 2.50288 & 0.07874 \\
$(\alpha=1)$ & 100 & 2.53168 & 0.03168 & 0.07473 & 2.50636 & 0.07230 \\
0.5 & & & & & & \\
& 20 & 0.52378 & 0.02378 & 0.01557 & 0.49759 & 0.01355 \\
& 40 & 0.51437 & 0.01437 & 0.00723 & 0.50151 & 0.00668 \\
& 60 & 0.50897 & 0.00897 & 0.00452 & 0.50049 & 0.00429 \\
& 80 & 0.50565 & 0.00565 & 0.00351 & 0.49933 & 0.00339 \\
& 100 & 0.50331 & 0.00331 & 0.00243 & 0.49827 & 0.00237 \\
\hline & & & & & & $($ Continued $)$ \\
& & & & & &
\end{tabular}


Table 9 MLE and UMVUE of $\beta$ when $\alpha$ is known for inverted Kumaraswamy distribution based on order statistics. (Continued)

\begin{tabular}{ccccccc}
\hline $\boldsymbol{\beta}$ & $\boldsymbol{n}$ & $\hat{\boldsymbol{\beta}}_{\text {MLE }}$ & $\boldsymbol{B i a s}_{(\text {MLE })}$ & $\boldsymbol{M S E}_{(\text {MLE })}$ & $\hat{\boldsymbol{\beta}}_{\text {UMVUE }}$ & $\boldsymbol{M S E}_{(\text {UMVUE })}$ \\
\hline 1 & 20 & 1.05252 & 0.05252 & 0.06152 & 0.99990 & 0.05303 \\
& 40 & 1.02846 & 0.02846 & 0.02938 & 1.00275 & 0.02716 \\
& 60 & 1.01387 & 0.01387 & 0.01828 & 0.99697 & 0.01750 \\
& 80 & 1.01335 & 0.01335 & 0.01518 & 1.00069 & 0.01351 \\
& 100 & 1.00906 & 0.00906 & 0.00980 & 0.99897 & 0.00953 \\
2.5 & 20 & 2.61420 & 0.11420 & 0.37656 & 2.48349 & 0.32835 \\
& 40 & 2.56035 & 0.06035 & 0.17277 & 2.49634 & 0.16079 \\
& 60 & 2.54435 & 0.04435 & 0.10973 & 2.50194 & 0.10420 \\
& 80 & 2.52224 & 0.02224 & 0.09390 & 2.49071 & 0.09117 \\
& 100 & 2.54406 & 0.04406 & 0.06967 & 2.51862 & 0.06672 \\
0.5 & & & & & & \\
& 20 & 0.53231 & 0.03231 & 0.01783 & 0.50570 & 0.01518 \\
& 40 & 0.51169 & 0.01169 & 0.00699 & 0.49890 & 0.00652 \\
& 60 & 0.50990 & 0.00990 & 0.00472 & 0.50140 & 0.00447 \\
& 80 & 0.50448 & 0.00448 & 0.00336 & 0.49817 & 0.00326 \\
1 & 100 & 0.50582 & 0.00582 & 0.00259 & 0.50076 & 0.00250 \\
& 20 & 1.03577 & 0.03577 & 0.05644 & 0.98398 & 0.05004 \\
& 40 & 1.01706 & 0.01706 & 0.02824 & 0.99164 & 0.02664 \\
& 60 & 1.01700 & 0.01700 & 0.01728 & 1.00005 & 0.01643 \\
& 80 & 1.01001 & 0.01001 & 0.01360 & 0.99738 & 0.01317 \\
& 100 & 1.00903 & 0.00903 & 0.00981 & 0.99894 & 0.00953 \\
& 20 & 2.63192 & 0.13192 & 0.36477 & 2.50033 & 0.31350 \\
& 40 & 2.59603 & 0.09603 & 0.19529 & 2.53113 & 0.17785 \\
& 60 & 2.53868 & 0.03868 & 0.12805 & 2.49637 & 0.11271 \\
& 80 & 2.52289 & 0.02289 & 0.07952 & 2.49136 & 0.07365 \\
& 100 & 2.52628 & 0.02628 & 0.06834 & 2.50102 & 0.06230 \\
\hline
\end{tabular}

Table 10 MLE and UMVUE of $\beta$ when $\alpha$ is known for inverted Kumaraswamy distribution based on lower records.

\begin{tabular}{ccccccc}
\hline $\boldsymbol{\beta}$ & $\boldsymbol{n}$ & $\hat{\boldsymbol{\beta}}_{\text {MLE }}$ & $\boldsymbol{B i a s}_{(\text {MLE })}$ & $\boldsymbol{M S E}_{(\text {MLE })}$ & $\hat{\boldsymbol{\beta}}_{\text {UMVUE }}$ & $\boldsymbol{M S E}_{(\text {UMVUE }}$ \\
\hline$(\alpha=0.5)$ & & & & & & \\
0.5 & 4 & 0.66557 & 0.16557 & 0.26088 & 0.49918 & 0.13132 \\
& 6 & 0.59808 & 0.09808 & 0.09449 & 0.49840 & 0.05894 \\
& 8 & 0.57595 & 0.07595 & 0.06511 & 0.50396 & 0.04545 \\
& 10 & 0.55709 & 0.05709 & 0.03852 & 0.50138 & 0.02856 \\
& 12 & 0.54336 & 0.04336 & 0.03096 & 0.49808 & 0.02444 \\
1 & 4 & 1.30753 & 0.30753 & 1.31251 & 0.98065 & 0.68546 \\
& 6 & 1.19347 & 0.19347 & 0.35580 & 0.99456 & 0.22112 \\
& 8 & 1.11630 & 0.11630 & 0.24408 & 0.97677 & 0.17706 \\
& 10 & 1.09644 & 0.09644 & 0.14771 & 0.98679 & 0.11228 \\
& 12 & 1.09222 & 0.09222 & 0.12473 & 1.00120 & 0.09766 \\
2.5 & 4 & 3.33735 & 0.83735 & 5.22589 & 2.50301 & 2.54518 \\
& 6 & 3.04286 & 0.54286 & 2.41545 & 2.53572 & 1.47402 \\
& 8 & 2.78516 & 0.28516 & 1.18280 & 2.43701 & 0.84729 \\
& 10 & 2.75911 & 0.25911 & 0.98954 & 2.49220 & 0.74293 \\
$(\alpha=1)$ & 12 & 2.70940 & 0.20940 & 0.68496 & 2.48362 & 0.53898 \\
0.5 & & & & & & \\
& 4 & 0.66773 & 0.16773 & 0.22474 & 0.50080 & 0.11059 \\
& 6 & 0.60390 & 0.10390 & 0.10984 & 0.50325 & 0.06879 \\
& 8 & 0.56442 & 0.06442 & 0.05583 & 0.49386 & 0.03961 \\
& 10 & 0.55428 & 0.05428 & 0.04078 & 0.49885 & 0.03065 \\
& 12 & 0.55223 & 0.05223 & 0.03381 & 0.50621 & 0.02616 \\
\hline
\end{tabular}


Table 10 MLE and UMVUE of $\beta$ when $\alpha$ is known for inverted Kumaraswamy distribution based on lower records. (Continued)

\begin{tabular}{|c|c|c|c|c|c|c|}
\hline$\beta$ & $n$ & $\hat{\boldsymbol{\beta}}_{M L E}$ & $\operatorname{Bias}_{(M L E)}$ & $M S E_{(M L E)}$ & $\hat{\boldsymbol{\beta}}_{U M V U E}$ & $M S E_{(U M V U E)}$ \\
\hline \multirow[t]{5}{*}{1} & 4 & 1.33507 & 0.33507 & 0.97265 & 1.00130 & 0.48396 \\
\hline & 6 & 1.21079 & 0.21079 & 0.39050 & 1.00899 & 0.24041 \\
\hline & 8 & 1.15320 & 0.15320 & 0.23482 & 1.00905 & 0.16190 \\
\hline & 10 & 1.11111 & 0.11111 & 0.16608 & 1.00000 & 0.12453 \\
\hline & 12 & 1.09891 & 0.09891 & 0.12318 & 1.00733 & 0.09534 \\
\hline \multirow[t]{5}{*}{2.5} & 4 & 3.08184 & 0.58184 & 3.86241 & 2.46138 & 1.89275 \\
\hline & 6 & 2.93978 & 0.43978 & 2.26822 & 2.44981 & 1.34336 \\
\hline & 8 & 2.90478 & 0.40478 & 1.46366 & 2.54169 & 0.99690 \\
\hline & 10 & 2.78098 & 0.28098 & 1.00504 & 2.50288 & 0.75014 \\
\hline & 12 & 2.73585 & 0.23585 & 0.84201 & 2.50786 & 0.66085 \\
\hline \multicolumn{7}{|c|}{$(\alpha=2.5)$} \\
\hline \multirow[t]{5}{*}{0.5} & 4 & 0.65761 & 0.15761 & 0.20665 & 0.49321 & 0.10231 \\
\hline & 6 & 0.60052 & 0.10052 & 0.09825 & 0.50043 & 0.06121 \\
\hline & 8 & 0.58375 & 0.08375 & 0.06552 & 0.51078 & 0.04491 \\
\hline & 10 & 0.54310 & 0.04310 & 0.03537 & 0.48879 & 0.02727 \\
\hline & 12 & 0.55120 & 0.05120 & 0.03410 & 0.50526 & 0.02648 \\
\hline \multirow[t]{5}{*}{1} & 4 & 1.33677 & 0.33677 & 0.99731 & 1.00257 & 0.49720 \\
\hline & 6 & 0.68309 & 0.18309 & 0.41968 & 0.51232 & 0.21737 \\
\hline & 8 & 0.57585 & 0.07585 & 0.06433 & 0.50387 & 0.04486 \\
\hline & 10 & 0.56139 & 0.06139 & 0.04769 & 0.50525 & 0.03560 \\
\hline & 12 & 0.54230 & 0.04230 & 0.02897 & 0.49710 & 0.02285 \\
\hline \multirow[t]{5}{*}{2.5} & 4 & 3.19019 & 0.69019 & 4.28578 & 2.54265 & 2.85432 \\
\hline & 6 & 2.96742 & 0.46742 & 2.41758 & 2.47285 & 1.52789 \\
\hline & 8 & 2.86216 & 0.36215 & 1.34216 & 2.50439 & 0.92719 \\
\hline & 10 & 2.80393 & 0.30393 & 0.98194 & 2.52354 & 0.75142 \\
\hline & 12 & 2.71570 & 0.21570 & 0.78274 & 2.48939 & 0.61874 \\
\hline
\end{tabular}

- From Tables 7 and 8 , one can easily see that for various configuration of parameters, biases and MSEs are decreasing as the sample size is increasing for order statistics and lower record case. Also it is noted at $(\alpha, \beta)=(0.5,1)$, MSEs of $\alpha$ are smaller than MSEs of $\beta$ and when $(\alpha, \beta)=(1,0.5)$, MSE $(\beta)>\operatorname{MSE}(\alpha)$ for all choices of sample sizes. Moreover, it is noted that order statistics performed better compared to lower records based on the simulated result.

- From Tables 9 and 10, it is evident that for all the parameter values and sample sizes, $M S E_{U M V U}<M S E_{M L}$, i.e., UMVUE are more adequate than MLE for both order statistics and lower records, and also UMVUE free from bias. Based on the simulated result, we can conclude that UMVUE perform better than MLE when one shape parameter is known and in this situation, use of UMVUE is recommended.

\section{CONCLUSION}

In this paper, we have obtained exact and explicit expression for single, product, and conditional moments of dgos from inverted Kumaraswamy distribution. The results are obtained in terms of Gauss hypergeometric and beta functions. Further, by adjusting the parameter values of $d g o s$, we obtained the moments of order statistics and lower record values. Based on these results, mean and variances of order statistics, lower record values, and dgos are calculated. We have also obtained MLE for all shape parameters and UMVUE for one shape parameter by taking another one is known as inverted Kumaraswamy distribution based on dgos. Based on the simulation result, we conclude that UMVUE perform better than MLE when one shape parameter is known and it is also noted that MSEs of MLE and UMVUE are decreasing as the sample size increases.

\section{ACKNOWLEDGMENT}

The authors would like to thanks the reviewers for reading the manuscript carefully, for detailed comments, and helpful suggestions which greatly helped to improve the manuscript.

\section{REFERENCES}

1. U. Kamps, A Concept of Generalized Order Statistics, B. G. Teubner, Rtuttgart, Germany, 1995.

2. P. Pawlas, D. Szynal, Demonstr. Math. 34 (2001), 353-358. 
3. M. Burkschat, E. Cramer, U. Kamps, Metron. 61 (2003), 13-26.

4. A.H. Khan, M.J.S. Khan, Pak. J. Stat. 28 (2012), 59-68.

5. P. Pawlas, D. Szynal, Commun. Stat. Theory Methods. 30 (2001), 739-746.

6. E. Cramer, U. Kamps, T. Rychlik, Stat. Probab. Lett. 59 (2002), 397-404.

7. M. Ahsanullah, Commun. Stat. Theory Methods. 33 (2004), 2921-2928.

8. H. Athar, H.M. Islam, M. Yaqub, J. Appl. Stat. Sci. 16 (2007), 37-46.

9. A.E.A. Ahmad, Commun. Stat. Theory Methods. 37 (2008), 1162-1172.

10. R.U. Khan, Z. Anwar, H. Athar, Aligarh J. Stat. 28 (2008), 37-45.

11. M.J.S. Khan, Z. Haque, M. Faizan, Aligarh J. Stat. 29 (2009), 107-119.

12. M. Burkschat, Commun. Stat. Theory Methods. 39 (2009), 311-326.

13. H.M. Barakat, M.E. Adll, Stat. Probab. Lett. 79 (2009), 1252-1259.

14. H. Athar, M. Faizan, Int. J. Stat. Sci. 11 (2011), 125-134.

15. F. Domma, G.G. Hamdeni, J. Stat. Theory Appl. 13 (2014), 222-234.

16. M.A.W. Mahmoud, Y. Abdel-Aty, N.M. Mohamed, G.G. Hamedani, J. Stat. Appl. Prob. 3 (2014), 189-199.

17. J. Saran, N. Pushkarna, R. Tiwari, J. Stat. Theory Appl. 14 (2015), 123-130.

18. M.J.S. Khan, M. Arshad, Am. J. Math. Manag. Sci. 35 (2016), 171-181.

19. M.J.S. Khan, S. Iqrar, Commun. Stat. Theory Methods. 48 (2019), 479-492.

20. M. Arshad, Q. Jamal, Am. J. Math. Manag. Sci. 38 (2019), 227-240.

21. P. Kumaraswamy, J. Hydrol. 46 (1980), 79-88.

22. S. Nadarajah, J. Hydrol. 348 (2008), 568-569.

23. M.C. Jones, Stat. Methodol. 6 (2009), 70-81.

24. M. Garg, Tamsui Oxford J. Inf. Math. Sci. 25 (2009), 153-166.

25. G.M., CordeiroM. De-Castro, J. Stat. Comput. Simul. 81 (2011), 883-898.

26. P.A. Mitnik, Commun. Stat. Theory Methods. 42 (2013), 741-755.

27. S.K. Safi, R.H. Ahmed, Statistical estimation based on generalized order statistics from Kumaraswamy distribution, in Proceedings of the 15th Applied Stochastic Models and Data Analysis (ASMDA), Matar, Barcelona, Spain, 2013, pp. 25-28.

28. M.M.S. El-Deen, G.R. AL-Dayian, A.A. El-Helbawy, Br. J. Math. Comput. Sci. 4 (2014), 1710-1743.

29. A.M. Abd AL-Fattah, A.A. EL-Helbawy, G.R. AL-Dayian, Pak. J. Stat. 33 (2017), 37-61.

30. R.M. Usman, M. Ahsan ul Haq, J. King Saud Univ.-Sci. 32 (2018), 356-365.

31. H. Reyad, F. Jamal, S. Othman, N. Yahia, Asian J. Math. 13 (2019), 1-15.

32. A.P. Prudnikov, Y.A. Brychkov, O.I. Marichev, Integeral and Series, vol. 1, Gordon and Breach Science Publishers, Amsterdam, Netherlands, 1992.

33. M.J.S. Khan, A. Sharma, M.I. Khan, S. Kumar, Int. J. Comput. Theor. Stat. 2 (2015), 107-115.

34. A.M. Mathai, R.K. Saxena, Generalized Hypergeometric Functions with Applications in Statistics and Physical Science, Lecture Notes in Mathematics, vol. 348, Springer-Verlag, Berlin, Germnay, 1973.

35. M. Habibullah, M. Ahsanullah, Commun. Stat. Theory Methods. 29 (2000), 1597-1609.

36. Z.F. Jaheen, Commun. Stat. Theory Methods. 34 (2005), 785-794.

37. I. Malinowska, P. Pawlas, D. Szynal, Linear Algebra Appl. 417 (2006), 150-162.

38. Z.A. Abo-eleneen, Math. Comput. Simul. 81 (2010), 26-36.

39. S. Abo-Elfotouh, M.N. Nassar, Int. J. Contemp. Math. Sci. 6 (2011), 1749-1760.

40. C. Kim, Appl. Math. Sci. 10 (2016), 2903-2919.

41. M.J.S. Khan, B. Khatoon, Ann. Data Sci. (2020), 525-545.

42. A.A. Zghoul, Statistica. 71 (2011), 355-365. 